\title{
Modelling of shear-induced lift for non-spherical point particles in arbitrary flows
}

\author{
Jure Ravnik1, Yan Cui ${ }^{2,3}$, Matjaž Hriberšek1, Paul Steinmann² \\ ${ }^{1}$ Faculty of Mechanical Engineering, University of Maribor \\ Smetanova 17, SI-2000 Maribor, Slovenia \\ jure.ravnik@um.si; matjaz.hribersek@um.si \\ ${ }^{2}$ Chair of Applied Mechanics, Friedrich-Alexander University Erlangen- Nuremberg \\ Paul-Gordan-Str. 3, D-91052 Erlangen, Germany \\ yan.cui@fau.de,paul.steinmann@fau.de \\ ${ }^{3}$ School of Mechanical Science and Engineering, Huazhong University of Science \& Technology
}

China

\section{Extended Abstract}

Simulation of dispersed phase flow in industry-standard commercial CFD software is done by using Euler-Euler or Euler-Lagrange approaches. When using the Euler-Lagrange approach, most commercial software is limited to the treatment of spherical particles and usually neglects the shear-induced lift force. However, it is known, that the vast majority of industrially interesting flows encompass non-spherical particles in shear flows. One example is the pulp and paper industry, where the papermaking process crucially depends on the transport of fibres in a liquid suspension.

The shear-induced lift force acting on particles moving at small, but finite Reynolds number (i.e. the so-called Saffmantype lift) has been studied for several decades. A sphere moving through a high viscous liquid in a simple shear flow was investigated first by Saffman resulting in the calculation of the lift force at small, but finite Reynolds numbers via singular perturbation methods. Later Saffman lift has been extended by generalising the simple shear flow to an arbitrary linear shear flow. Harper and Chang [1] have derived an expression for lift force acting on an arbitrarily-shaped 3D body in a linear onedirectional shear flow. At this conference, we will introduce a generalization of the shear-induced lift force, which is applicable for arbitrary shear and arbitrarily-shaped particles (rigid prolate ellipsoids in the present study), as presented in [2-4].

We propose a generic method to extend the shear-induced lift force models that were originally devised for linear shear flow to arbitrary flow conditions by performing two coordinate rotations. This method is applied to the computation of the shear-induced lift force acting on a prolate spheroidal particle moving in arbitrary non-uniform flow. We first consider lift force arising from the dominant streamwise flow shear and then move on to consider the influence of the non-streamwise flow shear on the lift force. By assuming that the particle slip velocity is parallel to the fluid velocity along the particle trajectory, the lift force model for a prolate spheroidal particle is proposed and is applicable in the flow cases with streamwise and non-streamwise flow shear. Finally, we propose a method to reduce the computationally expensive calculations, which involve two coordinate rotations for each particle for every time step.

We verify the shear lift force model for prolate spheroidal particles by simulating the movement of particles in Poiseuille flow. Furthermore, to validate the ability of the model to capture the lift component arising from non-streamwise flow shear, the lift force model is compared with established generalised Saffman-type lift models by simulating the motion of a particle in lid-driven cavity flow. The computational results demonstrate that the developed lift force model for prolate spheroidal particles is applicable to flow cases with streamwise and non-streamwise flow shear.

The developed model has been used to simulate fibre like particles in a turbulent channel flow, where the flow simulations were obtained via DNS. Additionally, we will present results of particle tracking in complex geometries (human lung model), where the flow field is obtained via RANS.

\section{References}

[1] E. Harper and I. Chang, "Maximum dissipation resulting from lift in a slow viscous shear flow," J. Fluid Mech., vol. 33, pp. 209-225, 1968. 
[2] Y. Cui, J. Ravnik, M. Hriberšek and P. Steinmann, "A novel model for the lift force acting on a prolate pheroidal particle in an arbitrary non-uniform flow. Part I. lift force due to the streamwise flow shear," International Journal of Multiphase Flow, vol. 104 , pp. 103-112, 2018

[3] Y. Cui, J. Ravnik, M. Hriberšek and P. Steinmann, P. "A novel model for the lift force acting on a prolate spheroidal particle in arbitrary non-uniform flow. Part II. lift force taking into account the non-streamwise flow shear," International Journal of Multiphase Flow, vol. 111 , pp. 232-240, 2019

[4] Y. Cui, J. Ravnik, M. Hriberšek and P. Steinmann, "Towards a unified shear-induced lift model for prolate spheroidal particles moving in arbitrary non-uniform flow," Computers \& Fluids, vol. 196, pp. 104323, 2020. 